\title{
Delay-Balanced Smart Repeaters for On-Chip Global Signaling
}

\author{
Roshan Weerasekera*, Dinesh Pamunuwa ${ }^{\dagger}$, Li-Rong Zheng* and Hannu Tenhunen* \\ * Department of Electronic, Software and Computer Systems, \\ KTH School for Information and Communication Technology, \\ ELECTRUM 229, 16440 Kista, Sweden. \\ Email: \{roshan,lrzheng,hannu\}@imit.kth.se \\ $\dagger$ Centre for Microsystems Engineering, Faculty of Applied Sciences \\ Lancaster University, Lancaster LA1 4YR, United Kingdom \\ Email: d.pamunuwa@lancaster.ac.uk
}

\begin{abstract}
In this paper we propose a smart driver, whose drive strength is dynamically altered depending on the relative bit pattern, by partitioning it into a Main Driver and Assistant Driver. For a higher effective load capacitance both drivers switch, while for a lower effective capacitance the assistant driver is quiet. It is shown that in an UMC $0.18 \mu \mathrm{m}$ technology the potential peak power saving, for typical global wire lengths, can be as much $18 \%$ with a $12 \%$ jitter reduction over a traditional repeater for a data rate of $1 G b / s$.
\end{abstract}

\section{INTRODUCTION}

Scaling of wires and tighter integration has resulted in signal integrity problems which only add to the interconnection woes; cross-talk between signal lines results in signal corruption and variable delay, depending on the respective switching patterns. A key technique in reducing propagation delay and signal degradation is repeater insertion. Although very effective and simple, this has an adverse effect on power consumption, and it has been estimated that over $50 \%$ of the power in a high performance microprocessor is dissipated by repeaters charging and discharging interconnects [1]. Further, over $90 \%$ of this power is concentrated in only $10 \%$ of the interconnects; i.e. those which are classed as global and run for a significant fraction of the die length.

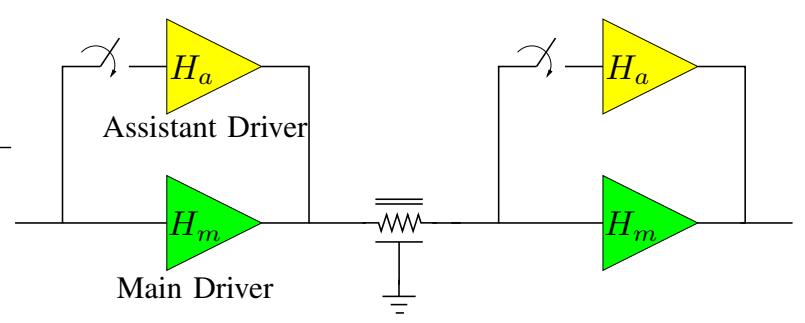

Fig. 1. Basic schematic of the proposed driver scheme

In this paper we propose a repeater that consumes less energy, and is suitable for exactly these kinds of global interconnections. It exploits the fact that in a parallel wire structure, the effective capacitance of a given wire is dynamic; i.e. it is a function of not only the physical geometry, but also the relative switching pattern described by the bits on the wire in question (the victim) and the adjacent wires (aggressors). With a traditional repeater, since the drive strength is static, the result is a spread of the propagation delay, with the repeater strength being essentially too much for every bit pattern other than the worst-case pattern. In the proposed repeater, the drive strength is dynamically altered depending on the relative bit pattern, by partitioning it into a Main Driver and Assistant Driver. For a higher effective load capacitance both drivers switch, while for a lower effective capacitance the assistant driver is quiet [2]. It is experimentally found that the potential peak power saving can be as as much $18 \%$ and the jitter reduction is about $12 \%$ over a traditional repeater for typical global wire lengths in UMC $0.18 \mu m$ technology.

The ramifications of the dynamically changing load in coupled interconnects have received a fair amount of attention in the literature. A comprehensive analysis of design considerations for repeater insertion in a bus structure with heavy coupling was presented in [3]. A scheme proposed in [4] staggers the repeaters so that opposing transitions only persist for the length of the offset between repeaters, and become bestcase patterns for the remainder, resulting in a delay reduction. Many innovative alternatives to the traditional repeater have also been proposed, such as the Transient Sensitive Accelerator (TSA) [5], Charge Recycling Technique (CRT) [6], Boosters [7], the TAGS receiver [8], the Aggressor-Aware Repeater [9], and the Capacitor Coupled Trigger and Accelerator combination [10]. Some of these use skewed inverters to trade-off noise margin for speed [5], [7], [8], while others consume more energy [9] and occupy a larger area [5], [7], [8] to produce a faster response.

In general, not only do these alternatives to traditional repeaters require much effort in circuit design similar to library cell design, but they also lack a clear high-level abstraction; in contrast, performance metrics such as delay and energy consumption can easily be quantified in terms of a few critical design parameters for the traditional inverting repeater [11], resulting in easy amalgamation in CAD flows $\mathrm{a}^{+}$diffamant levels of hierarchy from initial signal planning to det: 


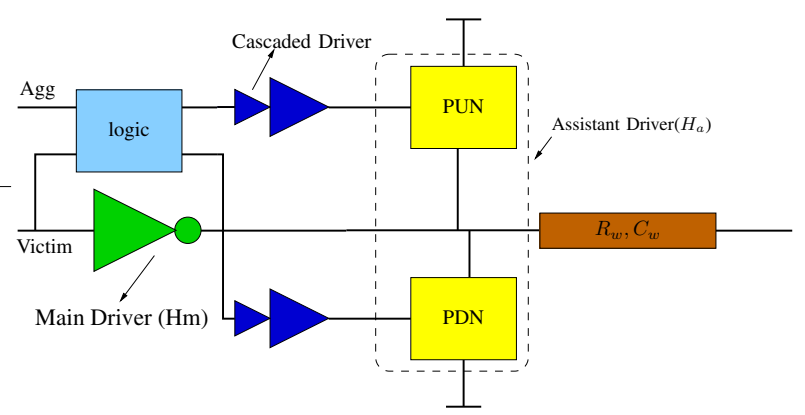

Fig. 2. Schematic of proposed SMART repeater, shown here only for two coupled nets

and route [12].

One of the main advantages of the repeater circuit proposed here is that the relatively minor increase in circuit complexity required to obtain the energy saving and delay equalization described above can be completely abstracted in the performance analysis. We present a design methodology similar to that for traditional single-wire inverting repeaters, including an $\mathrm{RC}$ equivalent circuit and closed-form expressions for the first-order approximation to the delay. Therefore this repeater can be very easily modeled in tasks such as delay calculation, signal integrity analysis and timing driven optimisation in any CAD flow for physical design.

The rest of this document is structured as follows. Section II describes the smart repeater circuit and circuit abstraction. The following section presents the design methodology. In the next section we present simulation results that validate the delay analysis. Then, we end with a discussion.

\begin{tabular}{|c|c|c|c|c|}
\hline \multirow[t]{2}{*}{ Group } & \multirow[t]{2}{*}{ Case } & \multicolumn{2}{|c|}{ Switching Event on } & \multirow{2}{*}{$\begin{array}{l}\text { Status of } \\
\text { the Assistant }\end{array}$} \\
\hline & & wire $i$ & wire $j$ & \\
\hline \multirow{2}{*}{1} & 1 & $\downarrow$ & $\downarrow$ & Off \\
\hline & 2 & $\uparrow$ & $\uparrow$ & Off \\
\hline \multirow{4}{*}{2} & 3 & $\overline{0}$ & $\overline{0}$ & $\overline{\text { Off }}$ \\
\hline & 4 & 0 & 1 & On \\
\hline & 5 & 1 & 0 & On \\
\hline & 6 & 1 & 1 & Off \\
\hline \multirow{4}{*}{3} & 7 & 0 & $\uparrow$ & Off \\
\hline & 8 & $\uparrow$ & 0 & Off \\
\hline & 9 & 0 & $\downarrow$ & Off \\
\hline & 10 & $\downarrow$ & 0 & On \\
\hline \multirow{4}{*}{4} & 11 & 1 & $\uparrow$ & Off \\
\hline & 12 & $\uparrow$ & 1 & On \\
\hline & 13 & 1 & $\downarrow$ & Off \\
\hline & 14 & $\downarrow$ & 1 & Off \\
\hline \multirow{2}{*}{5} & 15 & $\uparrow$ & $\downarrow$ & On \\
\hline & 16 & $\downarrow$ & $\uparrow$ & On \\
\hline
\end{tabular}

TABLE I

Status of Assistant DRIVER For POSSIBLE Switching ACTIVITIES ON THE LINES

\section{CONCEPT OF THE SMART DRIVER}

The variation of effective capacitance with the relative switching pattern introduces a spread in the arrival time at the far end of the wire, which is known as dynamic delay. To 20th International Conference on VLSI Design (VLSID'07)

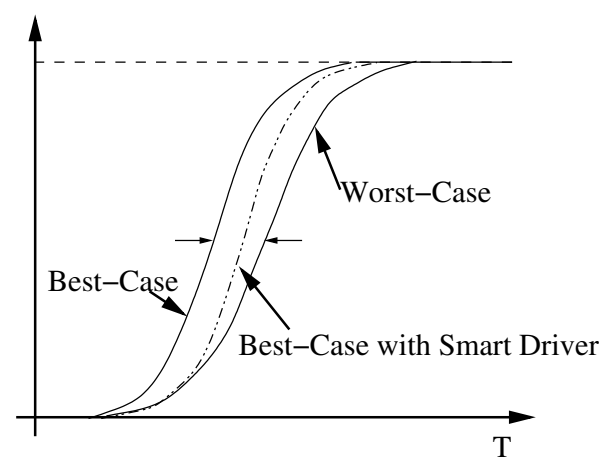

Fig. 3. Method of Jitter reduction using SMART driver

demonstrate this, a pair of coupled lines is used as a constituent unit for a bus. For two simultaneously switching lines, sixteen possible switching combinations can be identified (Refer Table I). These can be categorized into five different groups according to the effective capacitance as follows. Group 1: Both switch in the same direction; Group 2: Both lines are quiet (at 0 or 1); Group 3: One line is switching while the other is quiet at 0; Group 4: One line is switching while the other is quiet at 1; Group 5: The lines switch in opposite directions.

To ensure error-free operation, timing constraints have to be satisfied for the switching pattern that causes the worstcase delay, which are the $\uparrow \downarrow$ and $\downarrow \uparrow$ combinations. Since the effective load is highest for these patterns, the size of the buffer designed statically for the worst-case delay is much larger than would be necessary for the same timing requirements for other patterns [3]. Now this worst-case condition occurs only twice out of 16 possible input switching patterns, with a probability of $1 / 8$ for simultaneously switching lines if the transitions are equally distributed as in a random bit stream. For the 14 other cases, the wire is driven faster, which just translates to slack which typically cannot be used. The driver proposed here changes its drive strength depending on the neighbour's switching direction by using some simple logic. A basic schematic of the proposed SMART repeater is shown in Figure 2. If the switching pattern belongs to Groups 1,3 , or 4 , a single inverter (the Main driver) drives the interconnect. When a switching pattern in Group 5 occurs, another inverter (the Assistant) also drives the line, increasing the total drive strength appropriately.

In Figure 3 the curves with solid lines represent the output response of a conventional driver, for minimum effective capacitance (Best-Case) and maximum effective capacitance (Worst-Case). Our aim, with the SMART driver, is to delay the response for the best-case without affecting the worst-case, so that the variation in delay becomes as small as possible. In other words, the concept is to make the response slower with minimum effective capacitance, as the cycle delay has to be set to the worst-case delay anyway.

\section{A. Circuit Realization}

It is difficult to change the state of the assistant hofnmo the input completes its transition due to the inherent deli

COMPUTER SOCIETY 


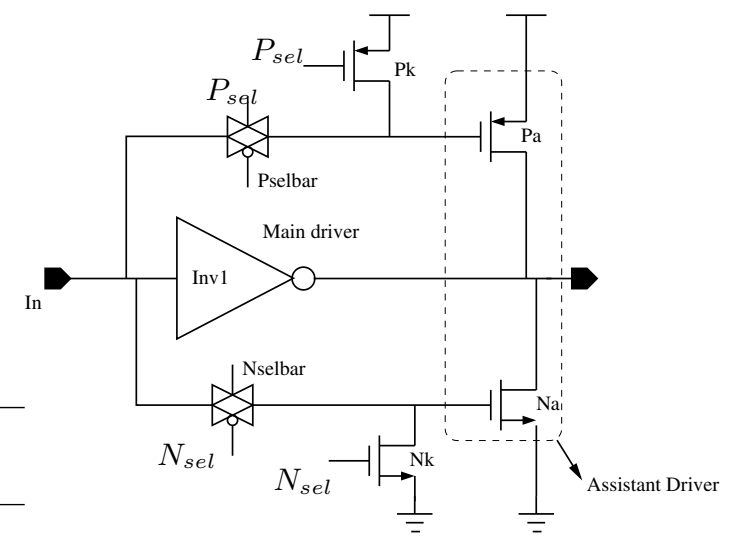

Fig. 4. Circuit Schematic of the proposed Smart driver

elements in the smart driver. Hence, in the implementation we anticipate and decide whether the next state would be a worstcase pattern or not. For example, assume that wire $i$ is at logic level zero(0) and wire $j$ is at logic level one(1) at this moment. One of the next states will be wire $i$ going to logic level one(1) and wire $j$ switching to logic level zero(0), which is a worstcase pattern described in Group 5. It is the same as with wire $i$ in logic level one(1) and wire $j$ being logic level zero(0) at the current state. If the wire $i$ at logic level 0 (1) and wire $j$ at logic level $1(0)$, the assistant is switched on. For all the other states the assistant is off. But due to this anticipation the assistant is on for not only the worst-case switching patterns, defined in Group 5, but also for two other patterns as well, i.e. case 10 and 12. The decision of making assistant on or off should be taken after the interconnect voltage reaches above threshold voltage and due to this reason the clock cycle has to be adjusted such that

$$
T_{\text {driver }} \leq T_{\text {logic }}<T_{\text {clk }}
$$

where $T_{\text {driver }}$ is the propagation delay of the driver, $T_{\text {logic }}$ is the delay of the selection logic circuit, and $T_{c l k}$ is the clock period. The output of the selection logic should be available just after the interconnect is driven, and this is the maximum clock rate that can be achieved with this proposed scheme. The lower bound of the inequality ensures that assistant driver is maintained until the output has crossed the threshold voltage $\left(V_{D D} / 2\right)$.

The transistor level schematic of the Smart driver is shown in the Figure 4. The transistors $\mathrm{Pa}$ and $\mathrm{Na}$ form the $\mathrm{As}$ sistant driver, where as the Inverter $I 1$ works as the Main Driver. Two transmission gates, TGp and TGn, are used to connect/disconnect the path from the signal input and the input of assistant driver. The transistors $P k$ and $N k$ will ensure that the assistant driver is off while the signal input path to the assistant is cut off, Otherwise the assistant driver will be just floating. The selection logic signals $P_{s e l}$ and $N_{s e l}$ are determined as:

$$
\begin{aligned}
& P_{\text {sel }}=\overline{A g g+\overline{\text { Victim }}} \\
& N_{\text {sel }}=\overline{\text { Agg. } \overline{\text { Victim }}}
\end{aligned}
$$

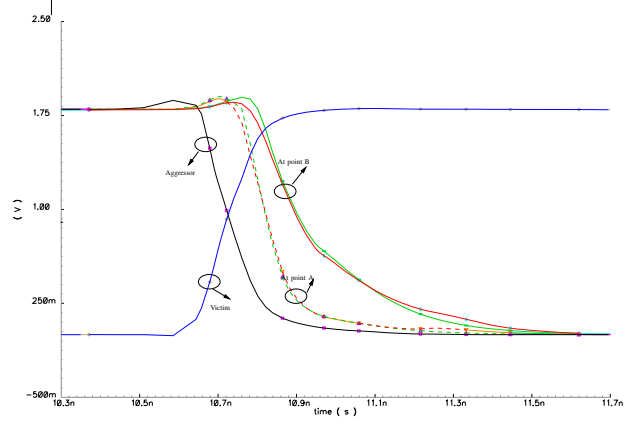

Fig. 5. When the both drivers are active: Aggressor and the Victim switches in different directions

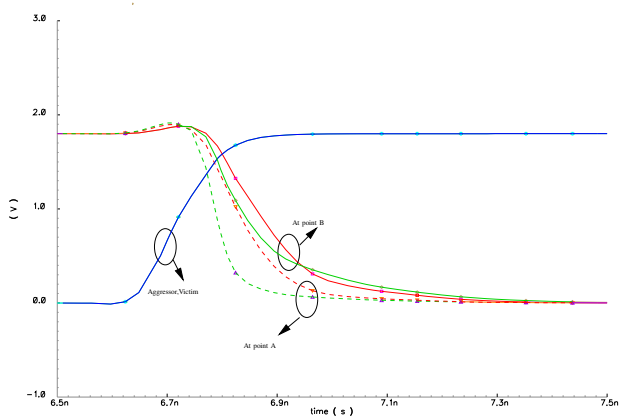

Fig. 6. Only when the main driver is active: Aggressor and the Victim switches in the same direction

When the victim input is at logic 0 and the aggressor is at logic 1 , the next victim stage would be logic 1 , and this might be a worst-case pattern if the aggressor too change its state. In this case we switched on $\mathrm{Na}$ and switched off $\mathrm{Pa}$ since $\mathrm{Pa}$ is not needed during this discharging time. This is achieved by setting $N a_{\text {sel }}=0$ and $P a_{\text {sel }}=0$.

Transmission gates is used as the switch to on/off the path between the wire input to the assistant driver, in order to reduce the path resistance. Cascaded two stage buffer is utilized to drive the transmission gate. Not only that the cascaded buffer delays the output of the selection logic so that it satisfy the condition mentioned in the Equation (1). If odd number of stages are needed in the cascaded buffer, selection logic has to be changed accordingly.

Figures 5 and 6 shows the simulations results at the far end and near end - points $\mathrm{A}$ and $\mathrm{B}$ in Figure 9 - of an $2.5 \mathrm{~mm}$ long wire driven by a smart repeater and a traditional repeater (inverter). When both the inputs, Aggressor and Victim, switches in the same direction the smart driver shows a delayed response, but the traditional driver shows a very faster response. But the far-end waveform the traditional repeater takes very a longer time to settle, but that for the smart driver is faster. When the victim and Aggressor switches in different directions, in this case victim switches upward and aggressor switches downward, both drivers show similar resnnncs smart driver has similar drive capability. 


\section{Delay Balancing With the SMART DRIVER}

\section{A. First-Order Delay Model}

The delay analysis for repeater insertion is carried out by characterizing a minimum sized inverter by an output resistance $R_{d m i n}$, in addition to the input gate capacitance $C_{g m i n}$ and output drain-diffusion capacitance $C_{d \min }$ already defined. Following notation is used as the wire electrical parameters: $R_{w}$ - total wire resistance, $C_{s}$ - total self wire (wire-to-ground) capacitance and $C_{c}$ - total interwire capacitance.

1) Delay Analysis with both Drivers Switching: With the linearisation of the driver, the equivalent circuit for one repeater segment can be shown to be the circuit in Figure 7 . Hence the $50 \%$ delay for the wire can be expressed as

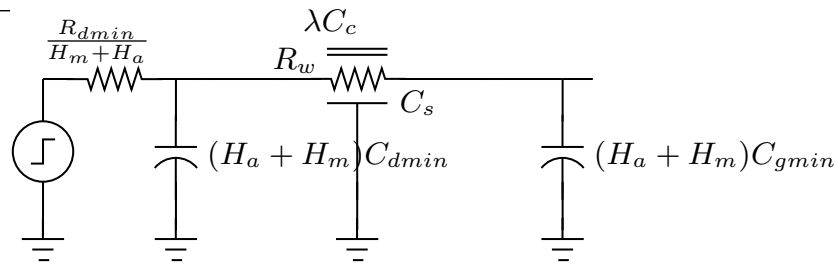

Fig. 7. Equivalent Circuit for the case when both drivers are switching

$$
\begin{aligned}
T_{M A} & =0.7 k\left(t_{\text {Dout }}+t_{D i n}\right)+\frac{0.7\left(t_{D W s}+\mu_{i} t_{D W c}\right)}{\left(H_{m}+H_{a}\right)} \\
& +0.7 t_{W D}\left(H_{m}+H_{a}\right)+0.4 \frac{t_{W s}}{k}+\frac{\lambda_{i} t_{W c}}{k}
\end{aligned}
$$

where $i=3$. Here $H_{m}$ and $H_{a}$ are the sizes of the Main and Assistant drivers respectively, $\lambda, \mu$ are the empherical switch factors ${ }^{1}$ proposed in [3], and to simplify the delay equation, the following time constants have been defined: $t_{\text {Dout }}=$ $R_{d m i n} C_{d m i n}, t_{D W s}=R_{d m i n} C_{s}, t_{D W c}=R_{d m i n} C_{c}, t_{D i n}=$ $R_{d m i n} C_{g m i n}, t_{W D}=R_{w} C_{g m i n}, t_{W s}=R_{w} C_{s}$ and $t_{W c}=$ $R_{w} C_{c}$.

2) Delay Analysis with the Assistant Quiet: When the Assistant driver is quiet while the Main driver is switching, the gate capacitance of the Assistant will not add to the load, as it is disconnected by a switch in which the input capacitance is negligible compared to the Assistant driver's input capacitance (see Figure 8). However the parasitic draindiffusion capacitance will always add to the load.

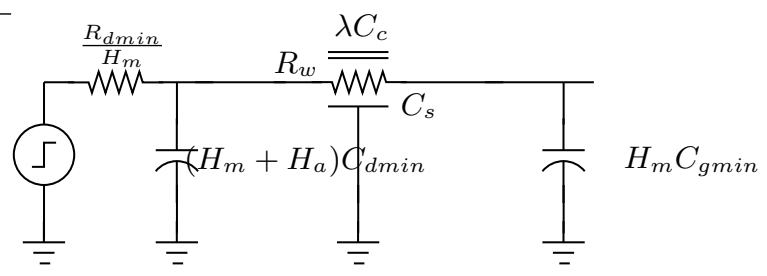

Fig. 8. Equivalent Circuit for the case when the Main driver is switching

${ }^{1}$ When one wire is switching and the other: switching in the same directions $\lambda_{0}=\mu_{0}=0$, is quiet either at zero or one $\lambda_{1}=0.57$ and $\mu_{0}=0.67$, switching opposite direction $\lambda_{2}=1.51$ and $\mu_{2}=2.2$.
The delay expression is now, with the time constants defined earlier:

$$
\begin{aligned}
T_{M} & =0.7 k\left[t_{\text {Dout }}\left(1+\frac{H_{a}}{H_{m}}\right)+t_{D i n}\right]+0.7 H_{m} t_{W D} \\
& +\frac{0.7\left(t_{D W s}+\mu_{i} t_{D W c}\right)}{H_{m}}+0.4 \frac{t_{W s}}{k}+\frac{\lambda_{i} t_{W c}}{k}
\end{aligned}
$$

where $i=1,2$

Equations (2) and (3) are the two principal delay equations of the SMART driver for its two states of Main and Assistant drivers switching, and Main driver switching while the Assistant driver is quiet.

\section{B. Sizing of the Drivers}

We obtain the optimal $H_{m}, H_{a}$ and $k$ values by deriving an expression for $\left(H_{m}+H_{a}\right)$ and $k$ by minimising $t_{M A}$.

$$
\begin{aligned}
H_{t}=H_{m_{-o p t}}+H_{a_{-o p t}} & =\sqrt{\frac{t_{D W s}+\mu_{i} t_{D W c}}{t_{W D}}} \\
k_{-o p t} & =\sqrt{\frac{\left.0.4 t_{W s}+\lambda_{i} t_{W c}\right)}{0.7\left(t_{D o u t}+t_{D i n}\right)}}
\end{aligned}
$$

Since the Assistant driver switches only for the worst-case switching pattern defined by Group 5 in Table I, the size of the Assistant driver, $H_{a}$, can be used to tune the delays for the other switching combinations defined by Groups 1 and 34. The expressions in (3) and (2) represent the delay for all these switching combinations. For clarity of explanation, say $T_{1}, T_{2}$ and $T_{3}$ are the wire delays for Groups 1, 3-4, and 5 respectively. Hence $T_{1}=\left.T_{M}\right|_{\lambda=0}, T_{2}=\left.T_{M}\right|_{\lambda=1}$ and $T_{3}=$ $T_{M A}$. Now increasing $H_{a}$ increases $T_{M}$ (see Figure ??), and hence $H_{a}$ can be sized so that either $T_{1}=T_{3}$ or $T_{2}=T_{3}$ ( $T_{1}=T_{2}=T_{3}$ is not possible because the relative delay variation between $T_{1}$ and $T_{2}$ is not a function of $H_{a}$ ).

The delay variation can be quantified as

$$
\Delta T=T_{M A}-T_{M} .
$$

By setting $\Delta T=0$, delay balancing can be achieved. Substituting for $T_{M A}$ and $T_{M}$ from (3) and (2) and using the relation $H_{m D B}=H_{t}-H_{a D B}$ the following quadratic for $H_{a D B}$ can be obtained.

$$
A H_{a D B}^{2}+B H_{a D B}+C=0
$$

where

$$
\begin{aligned}
A & =0.7 t_{W D} \\
B & =0.7\left[k t_{\text {Dout }}-t_{W D} H_{t}\right. \\
& \left.+\frac{t_{D W s}+2 t_{D W c}}{H_{t}}\right]+\frac{\left(\lambda_{2}-\lambda_{1}\right) t_{W c}}{k} \\
C & =0.7\left(\lambda_{3}-\lambda_{2}\right) t_{D W c}+\frac{0.4\left(\mu_{3}-\mu_{2}\right) t_{W C}}{k}
\end{aligned}
$$

Now sizing $H_{a D B}$ to equalise $T_{1}$ and $T_{3}$ results in $T_{2}$ being larger than $T_{3}$, which may not always be possible due to constraints on $T_{3}$, the worst-case delay. However amnlicine $T_{2}$ and $T_{3}$ does not result in any such adverse effec 
and $k$ can be calculated according to the strategy adopted. For example for optimal repeater insertion, $H_{t}$ can be calculated from Equation (4) and $k$ from Equation (5). Note also that $H_{a}$ is not a function of the wire length, but is solely depend on the crosstalk capacitance.

\begin{tabular}{l|l|l}
\hline \multirow{3}{*}{ Inverter (1X) } & $\left(R_{d \min } k \Omega\right)$ & 7.8 \\
\cline { 2 - 3 } & $C_{g \min }(f F)$ & 3.71 \\
\cline { 2 - 3 } & $C_{d \min }(f F)$ & 4.17 \\
\hline \hline \multirow{4}{*}{ Global Wire } & Width $(W)(n m)$ & 525 \\
\cline { 2 - 3 } & Aspect Ratio $(A R)$ & 2.1 \\
\cline { 2 - 3 } & $H(=2 W)(n m)$ & 1050 \\
\cline { 2 - 3 } & $k_{I L D}$ & 3.5 \\
\cline { 2 - 3 } & $r_{w}(\Omega / m m)$ & 38 \\
\cline { 2 - 3 } & $c_{s}(f F / m m)$ & 39 \\
\cline { 2 - 3 } & $c_{c}(f F / m m)$ & 101 \\
\hline
\end{tabular}

TABLE II

BUFFER CHARACTERISTICS FOR $0.18 \mu m$ AND Wire PARAMETERS

\section{Peak Power/Delay Comparison}

UMC $0.18-\mu m$ CMOS technology is used for the device models with a $V_{d d}$ of $1.8 \mathrm{~V}$, Cadence Spectre is used for all the simulations. A typical global metal layer is used for routing the bus, with a minimum pitch of $1050 \mathrm{~nm}$. The wire electrical parameters has obtained using the equation proposed in [13], and the inverter characteristics $R_{d m i n}, C_{d m i n}$, and $C_{g m i n}$, are experimentally determined as the simple method outlined in [14]. They are presented in Table II. Each interconnect wire segment is modeled as a $\pi-10$ capacitively coupled network. The two wires, representative unit for bus structures, has fed with two uniformly distributed pseudo random bit sequences(PRBS). The above bit sequence has a cycle time of $1 \mathrm{~ns}$ with a rise/fall time of $350 \mathrm{ps}$. In this particular design $T_{s e l}$ is $567 p s$ and that is the maximum cycle time that we can achieve using this scheme.

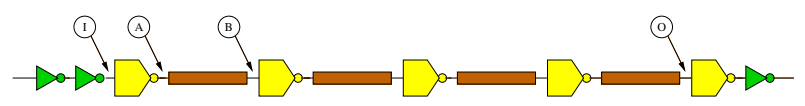

Fig. 9. Interconnect Link used for the Simulation - Total length is $10 \mathrm{~mm}$, and one segment is $2.5 \mathrm{~mm}$

For these parameters, the values for driver sizes and the number of repeaters needed for delay minimization and delaybalanced assistant are found using Equations (4), (5) and (6). They are presented in Table III:

\begin{tabular}{l|c|c|c|c|c|c}
\hline Case & $H_{t}$ & $k_{\text {opt }}$ & $H_{a}$ & $T_{\max }$ & $T_{\min }$ & $T_{\max }-T_{\min }$ \\
\hline \hline \multicolumn{6}{l}{ From Calculations ... } \\
\hline Traditional & 120 & 4 & - & $741 \mathrm{ps}$ & $488 \mathrm{ps}$ & $253 \mathrm{ps}$ \\
\hline Smart & 120 & 4 & 77 & $741 \mathrm{ps}$ & $600 \mathrm{ps}$ & $141 \mathrm{ps}$ \\
\hline From Simulations ... \\
\hline Traditional & 120 & 4 & - & $695 \mathrm{ps}$ & $483 \mathrm{ps}$ & $212 \mathrm{ps}$ \\
\hline Smart & 120 & 4 & 70 & $718 \mathrm{ps}$ & $531 \mathrm{ps}$ & $187 \mathrm{ps}$ \\
\hline
\end{tabular}

TABLE III

CAlculated values for optimal bufFer sizes, Related Delay and ENERGY
Simulations were carried out slightly increasing and decreasing the optimal buffer size, $H_{t}$, obtained such that it reduces the worst-case delay, but the reduction of the delay is not so pronounced and hence the estimated value itself has used for the simulations. For this repeater configuration the delays ${ }^{2}$ for different switching patterns are shown in the Figure 10. The $50 \%$ delay is measured from the input to the far-end of the last section (Point $O$ in Figure 9). It clearly depicts the idea of delay balancing, reducing the drive strength for low effective capacitance and increasing drive capability for higher effective capacitance. The variation of maximum and minimum delays with $H_{a}$ is shown in Figure 11 and found that $H_{a}$ should be in the range of 70-75 in order to have a minimum delay variation. In the case of the traditional driver the delay variation is $212 \mathrm{ps}$, but for the smart driver case it is $187 \mathrm{ps}$. With the help of the smart repeater technique the delay variation is reduced by about $12 \%$ and the peak power consumption is reduced by more or less 18\% (Peak power dissipation for the traditional case is $16 \mathrm{~mW}$ and that of smart driver is $13.1 \mathrm{~mW}$ ).

When $H_{a}$ is very small $10-20 \%$ of $H_{t}$ the delay variation is very much close that of a traditional driver, but as it increases, which in turn reduces the size of $H_{m}$, the delay variation is increased drastically (Figure 11).

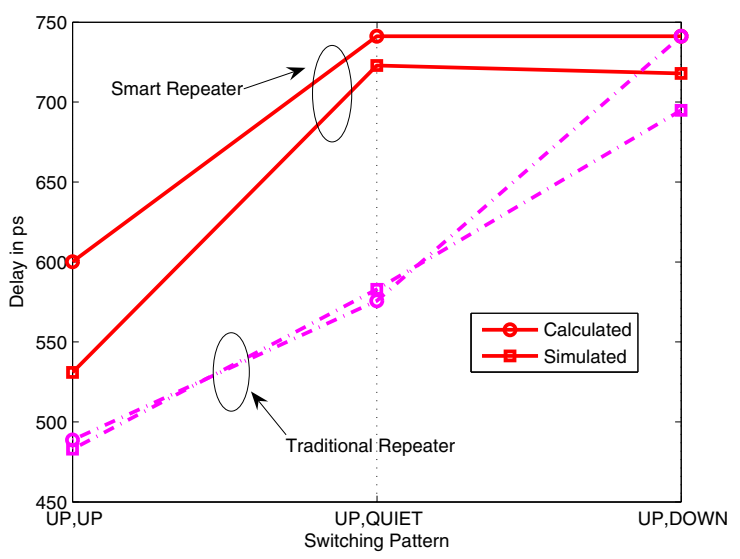

Fig. 10. Delay Comparison for two driver techniques

The delay model predicts that there might be a jitter reduction as much as $55 \%\left(=\frac{\Delta T_{\text {trad }}-\Delta T_{\text {smart }}}{\Delta T_{\text {trad }}}\right)$. Nevertheless, the average energy consumption per cycle for the smart driver and the selection logic combination is about $858 \mathrm{fJ}$, whereas the traditional driver consumes only $756 \mathrm{fJ}$, which is almost the same.

There is a slight increase in the peak crosstalk voltage with a smart driver compared to that of traditional but the peak crosstalk at the far-end is about $12 \%$ of $V_{d d}$, which is in the normal acceptable range of $20 \%$ of $V_{d d}$.

\footnotetext{
${ }^{2}$ The first order Elmore delay model presented in this work assumes that the input is a step, but in practice it is a finite ramp. In order to increase its accuracy, half of the rise time $\left(\frac{t_{r}}{2}\right)$ is added to the values comf and $T_{M A}$ 


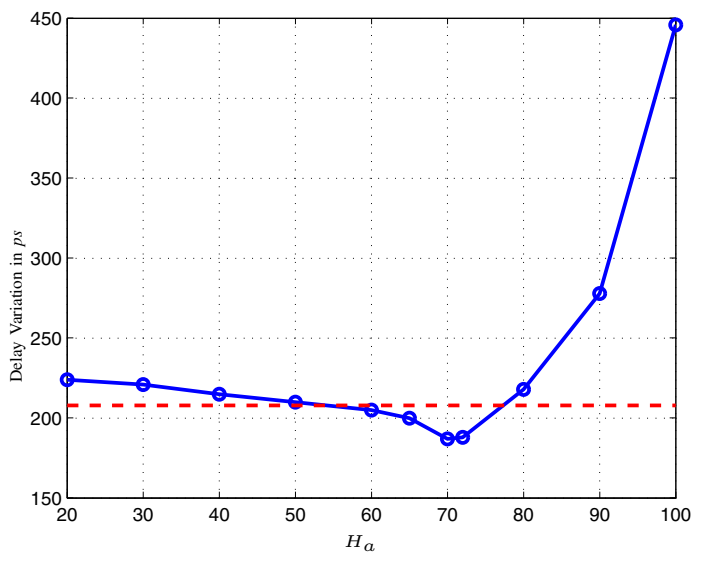

Fig. 11. The Variation of Delay Variation with Ha

\begin{tabular}{|c|c|c|}
\hline & Near End (A) & Far End (B) \\
\hline \hline Smart Repeater & $0.113 \mathrm{~V}$ & $0.213 \mathrm{~V}$ \\
\hline Traditional Repeater & $0.069 \mathrm{~V}$ & $0.206 \mathrm{~V}$ \\
\hline
\end{tabular}

TABLE IV

Maximum Crosstalk on a Quiet Line: Point A And B ARE The NEAR END AND FAR END

In order to demonstrate the relationship of coupling capacitance and the size of delay-balanced assistant buffer, for a variation of coupling capacitance the relative size of $H_{a}$ is found both in experimentally in circuit level and system level. Shown in Figure 12 is that relationship. However, the calculated and simulated values are not very close, but show similar characteristics.

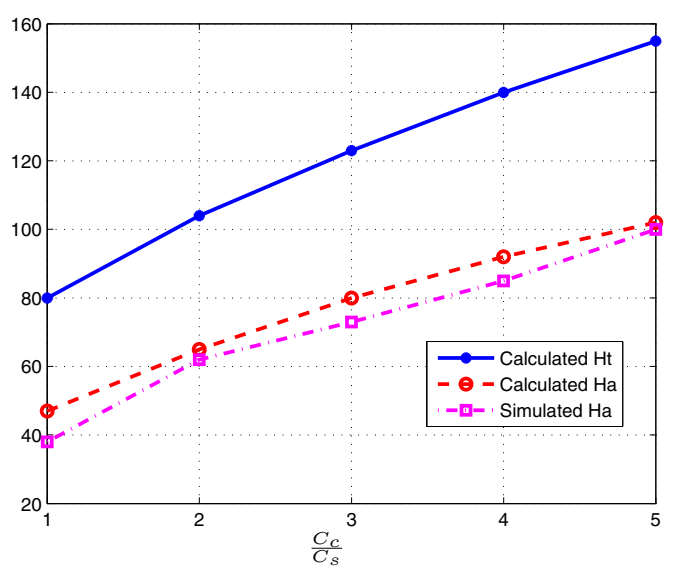

Fig. 12. Relative Size of Ha for different $\mathrm{Cc} / \mathrm{Cs}$

\section{CONCLUSIONS}

We have presented a detailed Elmore delay model for the SMART repeater and high level sizing for the Assistant and the Main drivers. A jitter reduction method called delay balancing technique is also presented. However, compared to a bus 20th International Conference on VLSI Design (VLSID'07) wire structure, the area for the extra gates are considerably small. With the same maximum delay as with traditional repeaters, SMART repeaters presented in this work shows $12 \%$ jitter reduction and a $18 \%$ peak power consumption. We conclude that SMART repeaters consumes less peak energy and reduce the data dependent jitter, with a area penalty but not a delay penalty. In future technologies since the transistors are less expensive than routing area, this would probably be an excellent replacement.

\section{REFERENCES}

[1] N. Magen, A. Kolodny, U. Weiser, and N. Shamir, "Interconnect-power dissipation in a microprocessor," in SLIP '04: Proceedings of the 2004 international workshop on System level interconnect prediction, 2004, pp. 7-13.

[2] R. Weerasekera, L.-R. Zheng, D. Pamunuwa, and H. Tenhunen, "Switching sensitive interconnect driver to combat dynamic delay in on-chip buses," in PATMOS 2005 Proceedings, Lecture Notes in Computer Science, vol. LNCS 3728, September 2005, pp. 277-285.

[3] D. Pamunuwa, L.-R. Zheng, and H. Tenhunen, "Maximizing throughput over parallel wire structures in the deep submicrometer regime," IEEE transactions on Very Large Scale Integration (VLSI) Systems, vol. 11, no. 2, pp. 224-243, April 2003.

[4] A. B. Kahng, S. Muddu, E. Sarto, and R. Sharma, "Interconnect tuning strategies for high-performance ics," in DATE '98: Proceedings of the conference on Design, automation and test in Europe, 1998, pp. 471478.

[5] T. Iima, M. Mizuno, T. Horiuchi, and M. Yamashina, "Capacitance coupling immune, transient sensitive accelerator for resistive interconnect signals of subquarter micron ulsi," IEEE Journal of Solid-State Circuits, vol. 31, no. 4, pp. 531-536, April 1996.

[6] P. Sotiriadis, T. Konstantakopoulos, and A. Chandrakasan, "Analysis and implementation of charge recycling for deep sub-micron buses," in ISLPED '01: Proceedings of the 2001 international symposium on Low power electronics and design, 2001, pp. 364-369.

[7] A. Nalamalpu, S. Sirinivasan, and W. P. Burleson, "Boosters for driving long on chip interconnects-design issues, interconnect synthesis, and comparison with repeaters," IEEE Transactions on Computer-Aided Design of Integrated Circuits and Systems, vol. 21, no. 1, pp. 50-62, January 2002.

[8] H. Kaul and D. Sylvester, "Low-power on-chip communication based on transition-aware global signaling(tags)," IEEE Transactions on Very Large Scale Integration (VLSI) Systems, vol. 12, no. 05, pp. 464-476, May 2004.

[9] A. Katoch, S. Jain, and M. Meijer, "Aggressor aware repeater circuits for improving on-chip bus performance and robustness," European SolidState Circuits, 2003. ESSCIRC '03. Conference on, pp. 261-264, Sep 2003.

[10] H.-Y. Huang and S.-L. Chen, "Interconnect accelerating techniques for sub-100-nm gigascale systems," IEEE Transactions on Very Large Scale Integration (VLSI) Systems, vol. 12, no. 11, pp. 1192-1200, November 2004.

[11] H. B. Backoglu, Circuits, Interconnections and Packaging for VLSI. Addison-Wesley, 1990.

[12] D. Pamunuwa, S. Elassaad, and H. Tenhunen, "Modeling delay and noise in arbitrarily coupled rc trees," IEEE Transactions on ComputerAided Design of Integrated Circuits and Systems, vol. 24, no. 11, pp. 1725-1739, November 2005.

[13] L.-R. Zheng, D. Pamunuwa, and H. Tenhunen, "Accurate a priori signal integrity estimation using a dynamic interconnect model for deep submicron vlsi design," in European Solid-State Circuits, 2000. ESSCIRC '00. Conference on, 2000, pp. 324-327.

[14] P. Caputa, R. Kallsten, and C. Svensson, "Capacitive crosstalk effects on on-chip interconnect latencies and data-rates," in NORCHIP Conference, 2005., 2005, pp. $281-284$. 